\title{
DISFUNÇÃO TÊMPORO-MANDIBULAR: ESTUDO INTRODUTÓRIO VISANDO ESTRUTURAÇÃO DE PRONTUÁRIO ODONTOLÓGICO
}

\section{TEMPOROMANDIBULAR JOINT DYSFUNCTION: INTRODUCTORY STUDY STRUCTURING DENTISTRY RECORDINGS}

\author{
Denise de Fátima Tomacheski ${ }^{1}$, Vânia Lílian Barboza ${ }^{1}$, \\ Márcio do Rocio Fernandes ${ }^{2}$, Fernando Fernandes ${ }^{3}$ \\ 1 Universidade Estadual de Ponta Grossa - UEPG, Campus em Uvaranas, Departamento \\ de Odontologia, Ponta Grossa, PR \\ 2 Rua João Manoel dos Santos Ribas, 823, Nova Rúsia, Ponta Grossa, PR; \\ e-mail: mfernandes@interponta.com.br \\ 3 Autor para contato: Universidade Estadual de Ponta Grossa - UEPG, Campus em \\ Uvaranas, Departamento de Odontologia, Ponta Grossa, PR, Brasil; (42) 220-3104; \\ e-mail: fernandfernandes@uol.com.br, ffernand@uepg.br
}

Recebido para publicação em 04/11/2003

Aceito para publicação em 19/07/2004

\section{RESUMO}

A disfunção têmporo-mandibular (DTM) e a dor orofacial são problemas que atingem grande parte da população. Muitos problemas estão relacionados à região da articulação têmporo-mandibular, como dores de cabeça e de ouvido, estalidos, dor facial, dentre outros. Compete ao cirurgião-dentista tratá-los. Com a criação da especialidade DTM e Dor Orofacial faz-se necessário sua abordagem nos currículos de graduação. Para o presente trabalho, foi aplicado um questionário aos acadêmicos do curso de Odontologia da UEPG no ano de 2002. O questionário proposto permitiu avaliar o grau de disfunção têmporo-mandibular nessa população avaliando-se a sua aplicabilidade. A partir dos resultados obtidos constatou-se que é grande a prevalência de DTM na população estudada e que é viável a aplicação do questionário proposto.

Palavras-chave: disfunção têmporo-mandibular, prontuário odontológico, oclusão

\begin{abstract}
Temporomandibular joint dysfunction (TJD) and orofacial pain are problems
\end{abstract}


that affect a great part of the population. Many problems are related to the area of the temporomandibular joint, such as headaches and earaches, clicks, facial pain and others. It is the task of the dentist to treat them. With the creation of the graduate course TJD and Orofacial Pain it became necessary to include the subject in the undergraduate curriculums also. In this study a questionnaire was developed and applied to the students of the undergraduate Dentistry program at UEPG in 2002. The results showed that the prevalence of TJD in that population is high. It also confirmed the applicability of the questionnaire.

Key words: temporomandibular joint dysfunction, dental recordings, occlusion

\section{Introdução}

O termo disfunção se refere à função que se desempenha de maneira anômala. A Disfunção Têmporo-Mandibular (DTM) corresponde, dessa forma, a ausência de normalidade nas funções do aparelho mastigatório (Maciel, 1998). Caracteriza-se por um conjunto de sinais e sintomas, como dores na região da articulação têmporo-mandibular (ATM), nos músculos da mastigação e em regiões radiadas da cabeça e do pescoço.

A DTM apresenta etiologia multifatorial e normalmente envolve uma abordagem interdisciplinar para seu tratamento (Rizzatti-Barbosa etal, 2000). Os fatores mais comumente relacionados são: maloclusão, fatores emocionais, ausências dentárias, mastigação unilateral, hábitos orais inadequados e outros ainda em estudo. Geralmente, apenas um fator isolado não é desencadeante da disfunção, mas sim a associação entre eles.

Essa doença, a DTM, é um mal que atinge parte da população, a qual normalmente não recebe informações sobre o que é desordem e como tratá-la. Geralmente médicos e cirurgiões dentistas não estão capacitados para diagnosticar a DTM. Não investigam a causa e tratam somente o sintoma, concorrendo assim para a não resolução definitiva do problema.

Este é um tema não muito explorado pela grande maioria dos cirurgiões dentistas, responsáveis pela área de DTM. Devido a essa deficiência as desordens vêm ganhando maior enfoque, respaldando assim a nova especialidade Disfunção Temporomandibular e Dor Orofacial. Tal temática motiva a implantação da disciplina DTM e Dor Orofacial nos cursos de graduação.

O presente trabalho teve por objetivo a coleta de dados a respeito da incidência de DTM entre os acadêmicos do curso de Odontologia matriculados no $1^{\circ}, 2^{\circ}, 3^{\circ}, 4^{\circ}$ e $5^{\circ}$ ano, da Universidade Estadual de Ponta Grossa (UEPG) e a utilização do prontuário ora proposto para, dessa forma, avaliarmos a sua aplicabilidade.

\section{Revisão de literatura}

A disfunção temporomandibular, segundo algumas definições, seria qualquer desarmonia que ocorre nas relações morfofuncionais: dos dentes, suas estruturas de suporte; maxilares; articulações temporomandibulares; músculos da mastigação; músculos dos lábios, língua, pescoço e os suprimentos vasculares e neurais das estruturas supracitadas. Em algumas outras definições está incluída também a idéia da existência de uma ruptura intrínseca das funções normais e ótimas do sistema, de forma que uma inter-relação harmoniosa dos vários órgãos componentes não mais existe (Santos Jr, 1987).

Diversos fatores podem estar relacionados à 
etiologia da DTM, sendo que dentre eles estão: maloclusão, falta de dentes, restaurações ou próteses mal adaptadas, mastigação unilateral, hábitos bucais inadequados, má-postura, tensão emocional, estresse, patologia ou trauma na articulação, fatores sistêmicos entre outros (Meira, 2002). É claro que muitas vezes esses fatores estão associados e os fatores que determinarão se o paciente apresenta ou não a desordem serão a tolerância fisiológica e a tolerância estrutural do indivíduo (Okeson, 1992).

Existem muitos sinais e sintomas que podem estar relacionadas à DTM. Dentre os mais comuns está a dor nos músculos da mastigação e/ou na ATM (Alves, 2002).

Quando há dor, devemos obter informações sobre sua localização, comportamento, tipo, duração e intensidade. A dor pode ser localizada quando o paciente aponta a área específica, ou pode aparecer como uma grande área mal definida. O local pode ser sempre o mesmo ou a dor pode estar irradiada. $\mathrm{O}$ comportamento da dor é avaliado através do relato do paciente quanto aos episódios em que ela ocorre: se a dor é constante ou intermitente, se ocorrem surtos ou períodos de dor e se há fatores que intensificam ou aliviam a dor. O tipo de dor pode ser definido como difusa ou aguda. A duração refere-se tanto à extensão do episódio (dias, semanas ou meses), quanto à permanência da dor durante um episódio (se ela não for constante). A intensidade é definida pelo paciente através de uma escala; como o grau de percepção varia de pessoa para pessoa, não deve haver índice único para a avaliação da dor (Okeson, 1992).

Segundo Santos Jr (1987), os sinais e sintomas relacionados às desordens temporomandibulares são: sons (estalidos ou clicking e crepitações), ressaltos articulares, subluxação, dor, limitações ou restrições nos movimentos mandibulares e fatores sistêmicos, como osteoartrite e artrite reumatóide. O autor ainda afirma que a dor na articulação é usualmente acompanhada por efeitos autonômicos, como mudanças na pressão arterial, pulsação, náusea e vômito.

Meira (2002) relata que os sinais e sintomas associados à DTM são: dor intra-articular, espasmo muscular, dor intra-articular combinada com espasmos musculares, dor reflexa, dor na abertura e fechamento da mandíbula, dor irradiada na área temporal, masseteriana ou infra-orbital; crepitação, dor ou zumbido no ouvido; dor irradiada no pescoço; dor de cabeça crônica; sensação de tamponamento do ouvido; xerostomia, entre outros.

Os pacientes relatam sons ou ruídos percebidos por eles na região da articulação. O estalido articular é um som único, seco, de curta duração que pode ocorrer durante a abertura e o fechamento mandibular. Está freqüentemente relacionado a problemas no disco articular. A crepitação é um som múltiplo e acompanha praticamente todo o trajeto condilar, indica provavelmente lesão do disco e o barulho é característico de contato ósseo do côndilo com a eminência articular. A crepitação é comum nas doenças degenerativas (Ramfjord e Ash, 1984).

A vertigem tem sido produzida, também, por uma área de desencadeamento miofacial, no músculo esternocleidomastoideo, de modo que pode haver alguma relação entre a vertigem e os espasmos musculares disfuncionais no sistema mastigador (Ramfjord e Ash, 1984).

Cefaléias e dor, zumbido ou tamponamento do ouvido, podem também estar relacionadas à DTM (Alves, 2002).

A dor de cabeça também compreende um sintoma associado à DTM (Okeson, 1992). Estimase que $80 \%$ de todas as dores de cabeça relatadas estão associadas a fontes musculares. Tem-se então que as atividades musculares da cabeça e do pescoço provavelmente desempenham um papel importante na etiologia de muitas dores de cabeça. Sendo assim, o tratamento direcionado a diminuir a hiperatividade muscular pode ter efeito significante na redução da dor de cabeça.

A dor de cabeça é provavelmente o sintoma mais comum e a queixa mais relatada dentre os sintomas da DTM. Numerosos fatores podem causar ou contribuir para o aparecimento das dores de cabeça, contudo, uma porcentagem significativa dessas dores pode estar relacionada com atividades musculares. Há relatos em que as alterações do tônus muscular podem ser responsáveis por 80 a $90 \%$ de todas as dores de cabeça (Maciel, 1998).

As causas da dor de cabeça podem ser: constante excitação do sistema nervoso simpático e do mecanismo de resposta hormonal ao estresse; macrotrauma, mastigação unilateral, apertamento dental, bruxismo, alterações musculares decorrentes de 
interferência oclusal e distúrbios no sistema circulatório do músculo (Garcia e Sousa, 1998).

É comum pacientes com dor na ATM também se queixarem de dor no ouvido. O ouvido encontra-se contido no osso temporal e relaciona-se com o côndilo mandibular, separado deste apenas pela parede timpânica. A proximidade do ouvido com a ATM e os músculos da mastigação como também suas inervações comuns no trigêmio, criam uma condição freqüente de dor reflexa (Maciel, 1998).

Otalgia sem causas orgânicas é um sintoma comum em pacientes com disfunção temporo-
mandibular(DTM), ainda que a etiologia seja controversa. Investigações da influência do tratamento da DTM por conta da otalgia são escassos (Keersmaekers et al, 1996).

\section{Metodologia}

Para avaliar-se o grau de DTM entre os estudantes, foi utilizado questionário proposto por Maciel (2002).

Questionário para determinar o índice de disfunção

1- Tem algum ruído no ouvido (zumbidos, tinidos, etc...)?

2- Tem alguma alteração na capacidade auditiva?

3- Tem alguma sensação ocasional de tamponamento auditivo?

4- Tem dificuldade para dormir?

5- Tem hábito de apertar ou ranger os dentes?

6- Tem os músculos masseteres hipertrofiados?

7- Tem ou teve alguma sensação de vertigem, tontura ou náusea?

8- Tem queixa de dores de cabeça, pescoço, ouvido com freqüência?

9- Tem tido fraturas de dentes e/ou restaurações?

10- Você se considera uma pessoa tensa, nervosa ou ansiosa? Em que grau (escala de 0 a 10)?

O questionário constituiu-se de dez perguntas objetivas que apontavam a presença da DTM. Cada pergunta tinha como opção de resposta as alternativas SIM, ÀS VEZES e NÃO, valoráveis conforme a Tabela 1. Na última pergunta, o valor obtido foi somado ao restante.

Tabela 1 - Valores atribuídos às respostas permitidas pelo questionário.

\begin{tabular}{lc}
\hline Respostas & Valores atribuídos \\
\hline SIM & 10 \\
ÀS VEZES & 5 \\
NÃO & 0 \\
\hline
\end{tabular}

Na análise das respostas, utilizou-se o índice para determinação do grau de DTM (Tabela 2).
Tabela 2 - Classificação da amostra segundo o grau de DTM.

\begin{tabular}{ll}
\hline Índice de D.T.M. & Classificação da D.T.M. \\
\hline $0-15$ & não portador de D.T.M. \\
$20-40$ & portador de D.T.M. leve \\
$45-65$ & portador de D.T.M. moderada \\
$70-100$ & portador de D.T.M. severa \\
\hline
\end{tabular}

Adotou-se o estudo quantitativo na análise dos dados e os mesmos receberam tratamento estatístico de forma descritiva e inferencial. Na análise descritiva utilizaram-se figuras (diagramas em barras). Na análise inferencial usaram-se porcentagens numéricas de ocorrência dos fenômenos colhidos. 


\section{Resultados}

Os resultados deste trabalho foram obtidos através da análise do questionário empregado com tal desiderato.

Num universo de 318 acadêmicos matriculados no curso de Odontologia da UEPG, 271 foram respondentes do questionário proposto. No Gráfico 1, observa-se o número de alunos matriculados em cada série e os que responderam ao questionário. No $1 .^{\circ}$ ano, de 63 matriculados, 57 responderam; no 2. ${ }^{\circ}$ ano, de 59 matriculados, 49 responderam; no $3 .^{\circ}$ ano, de 69 matriculados, 57 responderam; no $4 .^{\circ}$ ano, de 59 matriculados, 45 responderam; e no $5 .^{\circ}$ ano, de 68 matriculados, 64 responderam.

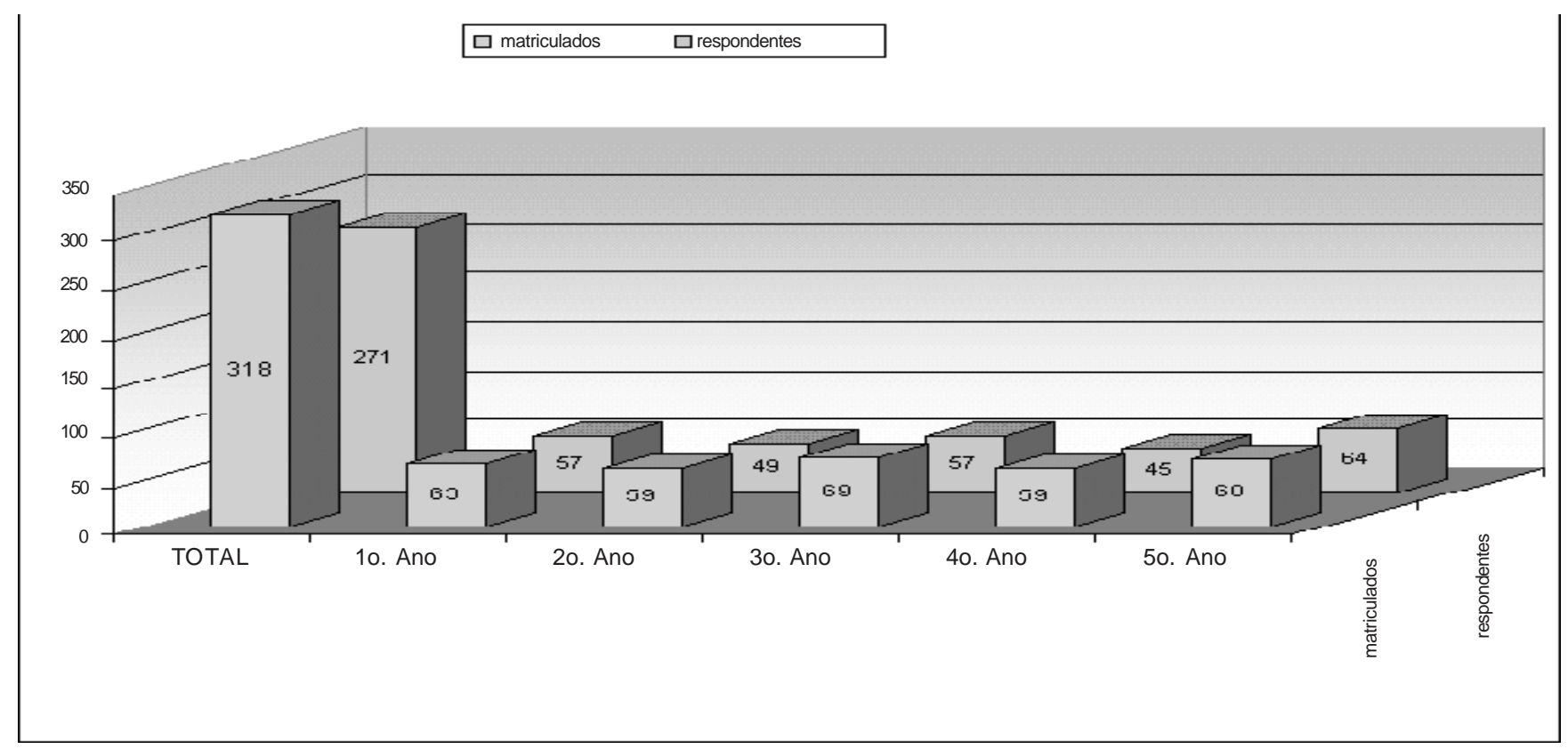

Gráfico 1 - Proporção alunos matriculados / alunos respondentes.

A seguir, o Gráfico 2 (Incidência de DTM na amostra), mostra a distribuição e a severidade da DTM, de acordo com o sexo. Com base no índice para determinação de disfunção empregado, observou-se no sexo feminino um percentual de $53,76 \%$ de portadoras de DTM leve, 6,87\% portadoras de DTM moderada e 39,37\% de não portadoras de DTM. No sexo masculino, 35,14\% eram portadores de DTM leve, 2,70\% portadores de DTM moderada e 62,16\% não eram portadores de DTM. Analisando o total de alunos entrevistados, os percentuais de pessoas não portadoras de DTM e portadoras de DTM leve foram próximos, respectivamente $48,71 \%$ e $46,12 \%$. O total de portadores de DTM moderada foi de 5,17\%. Não foi encontrado nenhum caso de DTM severo. 


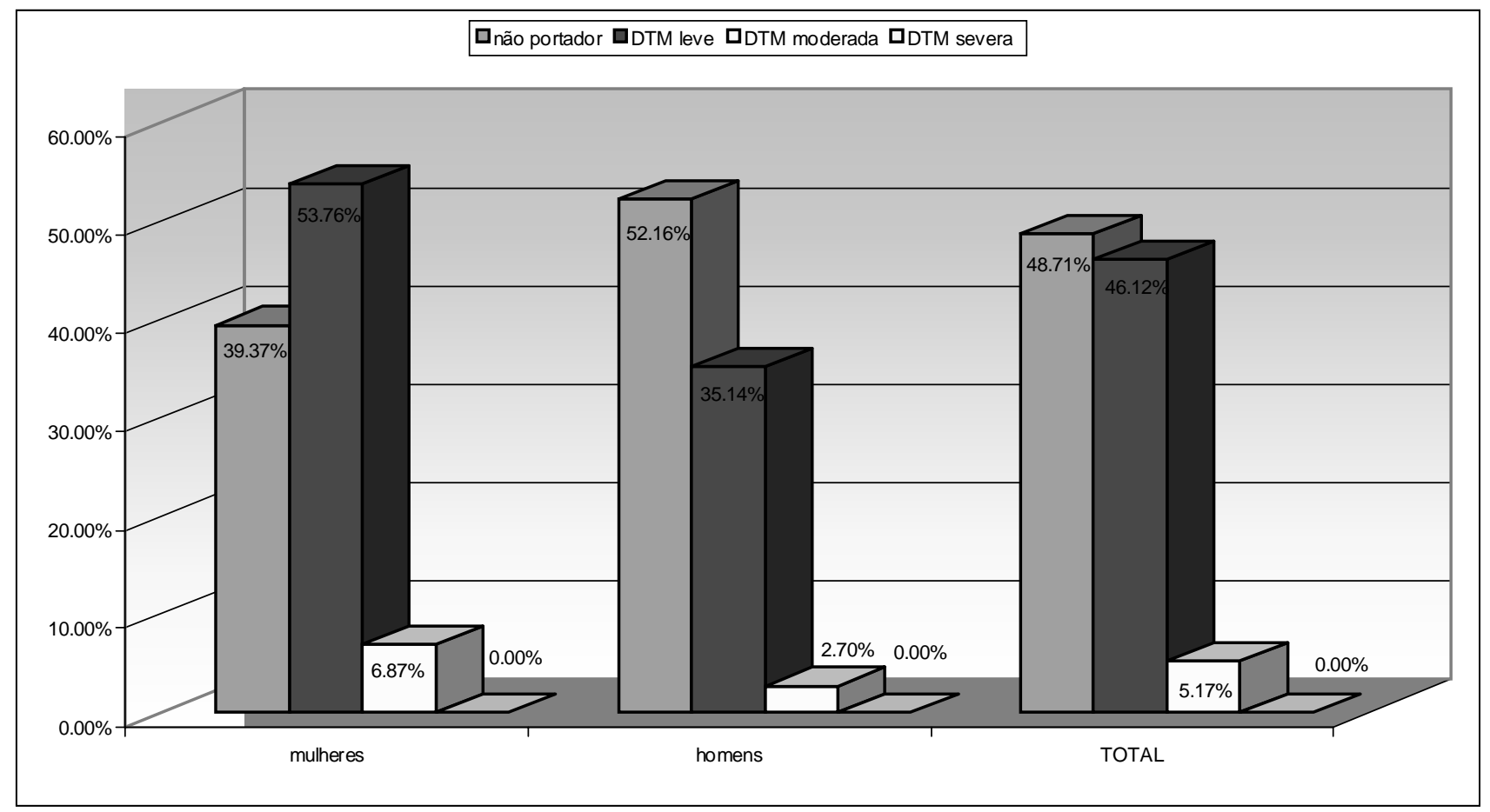

Gráfico 2 - Incidência de DTM na amostra.

Através do questionário utilizado foi avaliado o grau de disfunção na amostra (Gráfico 3 - Respostas ao questionário para determinar índice de disfunção). Observando as respostas obtidas, constatou-se:

- na questão 1 "Tem queixa de dor de cabeça, pescoço, ouvido com freqüência?” os índices de resposta obtidos foram 71,95\% não, 20,29\% às vezes e $7,74 \%$ sim;

- na questão 2 “Tem algum ruído no ouvido (zumbidos, tinidos, etc...)?” 80,44\% responderam não, $16,60 \%$ às vezes e $2,95 \%$ sim;

- na questão 3 “Tem ou teve alguma sensação de vertigem tontura ou náusea?” 64,20\% responderam não, $19,92 \%$ às vezes, e 5,86\% sim;

- na questão 4 "Tem alguma alteração na capacidade auditiva?” 88,56\% responderam não, $7,38 \%$ às vezes e $4,05 \%$ sim;

- na questão 5 “Tem alguma sensação de tampo- namento auditivo?” 63,83\% responderam não, $23,24 \%$ às vezes e $12,91 \%$ sim;

- na questão 6 "Tem hábito de apertar ou ranger os dentes?” 60,88\% responderam não, 16,97\% às vezes e $22,14 \%$ sim;

- na questão 7 “Tem tido fraturas de dentes e/ou restaurações?” 81,91\% responderam não, 4,79\% às vezes e $13,28 \%$ sim;

- na questão 8 "Tem os músculos masseteres hipertrofiados?” 92,98\% responderam sim, 0,73\% às vezes e 6,64\% sim;

- na questão 9 “Tem dificuldade para dormir?” $88,19 \%$ responderam não, $8,11 \%$ às vezes e 3,69\% sim;

- na questão 10 "Você se considera uma pessoa tensa, nervosa ou ansiosa?” $11,44 \%$ responderam não, 26,94\% e 61,62\% sim. 


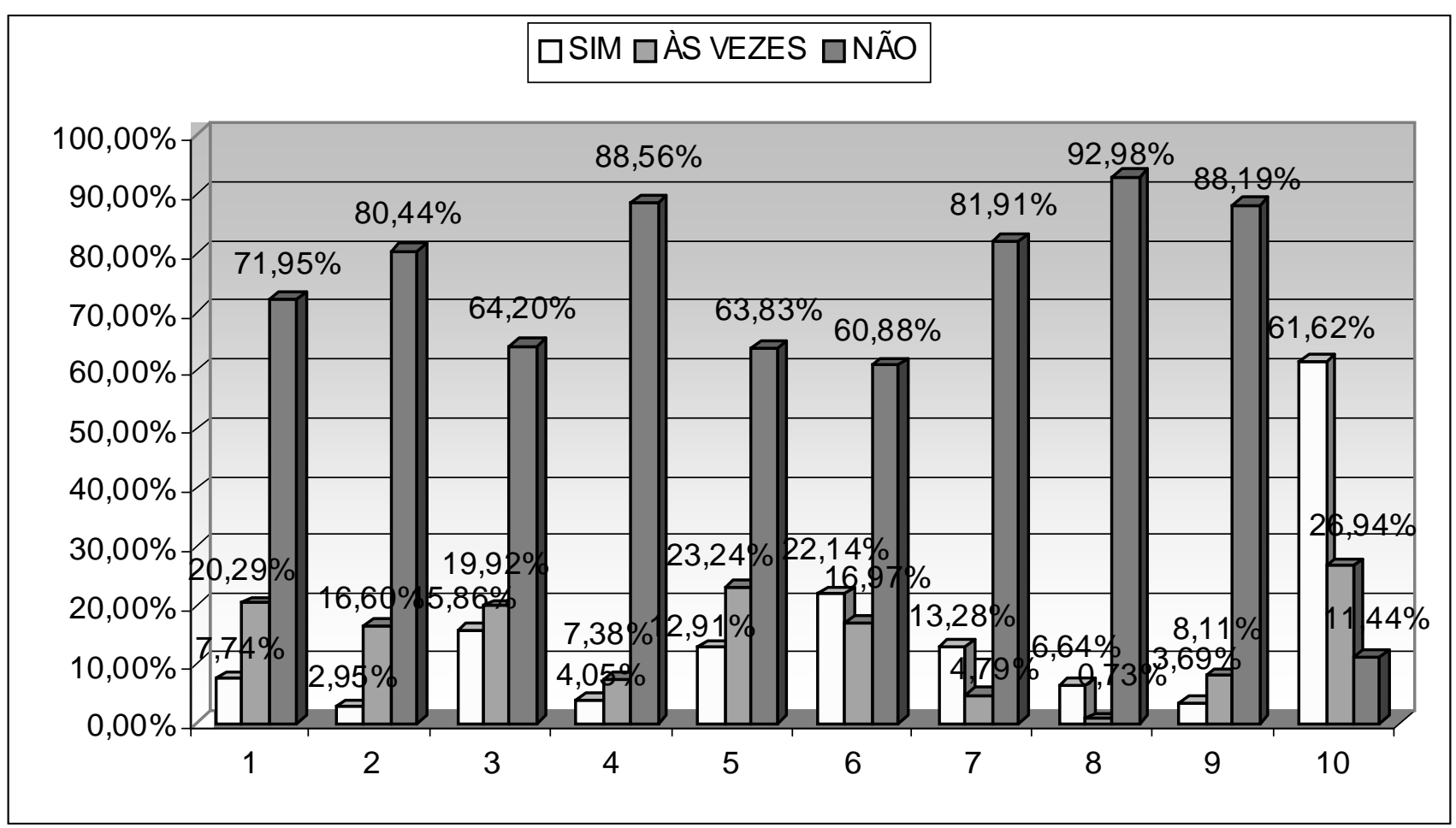

Gráfico 3 - Respostas ao questionário para determinar índice de disfunção.

\section{Discussão}

O índice anamnésico empregado mostrou-se altamente sensível para avaliar o grau de DTM na população estudada. Com este índice é possível a obtenção de um diagnóstico precoce da DTM. Okeson (1992) afirma que para se conseguir um diagnóstico preciso da desordem temporomandibular alguns fatores devem ser analisados, como o histórico da DTM, pois no diagnóstico da dor cerca de 70 a $80 \%$ da informação para conseguirmos identificar a desordem virá dele, sendo que o exame clínico contribuirá com uma parte menor.

Estudando cuidadosamente os resultados obtidos nesta investigação, destaca-se o fato de as mulheres apresentarem maior prevalência de DTM leve e moderada que os homens. Moresca e Urias (2001) constataram que a maior freqüência de ruídos articulares nas mulheres demonstra a maior predisposição do sexo feminino em desenvolver problemas na ATM. Esse achado está de acordo também com Okeson (1992), que relatou existir uma flacidez generalizada dos tecidos no sexo feminino, que pode ser devida ao aumento do nível de estrógenos. As articulações das mulheres são geralmente mais flexíveis e frouxas que as dos homens. Gage et al (1995) relatou que foi encontrado aproximadamente o dobro de colágeno tipo III no ligamento posterior da ATM em mulheres do que em homens. Esse fato indica que esses tecidos, nas mulheres, são menos capazes de suportar pressão funcional. No entender de Rieder et al (1983), a mulher é mais estressada do que o homem e apresenta um maior índice de doenças com envolvimento psicossomático.

A queixa de dores de cabeça, pescoço e ouvido freqüentes (QUESTÃO 1) foi relatada por 7,74\% da população estudada, sendo que $20,29 \%$ apresentavam ocasionalmente esta sintomatologia.

O pequeno percentual de respostas afirmativas é contraria o que se encontra na literatura onde, a dor de cabeça é citada como sendo o principal sintoma associado a DTM (Okeson, 1992; Maciel, 1998; Garcia e Souza, 1998). Uma porcentagem significativa dessas dores pode estar relacionada às atividades musculares. Garcia e Souza (1998) analisaram 34 pacientes portadores de DTM e constataram que 53,94\% apresentavam dor de cabeça, sendo que o sintoma mais comum entre esses pacientes foi o mio- 
espasmo. Verificaram ainda que após orientação, instalação de placa reposicionadora e ajuste oclusal, a dor de cabeça era eliminada.

Outro sintoma questionado foi a dor no pescoço, que pode estar presente em indivíduos portadores de disfunção. Um ponto álgico nos músculos da região cervical pode desencadear dor reflexa na região temporal (Okeson, 1992; Maciel, 1998).

A dor no ouvido pode estar presente na disfunção devido à proximidade existente entre o ouvido e a ATM e os músculos da mastigação, pois estas estruturas apresentam inervações comuns no trigêmio, criando uma situação freqüente de dor reflexa (Maciel, 1998).

Ruídos no ouvido, como tinidos e zumbidos (QUESTÃO 2) tiveram 2,95\% de respostas afirmativas. Os ruídos articulares (estalidos e crepitações) estão normalmente associados a problemas no disco articular, porém apenas a presença do estalido articular por vários anos sem o relato de dor não determina a progressão do quadro de disfunção, não necessitando, portanto, de tratamento (Ramfjord e Ash, 1984; Santos Jr, 1987).

Vertigem, tontura e náusea (QUESTÃO 3) apareceram em 15,86\% das respostas. Um paciente com disfunção em episódios de dor, pode desencadear efeitos autonômicos, como alteração de pressão, náusea e vômito (Ramfjord e Ash, 1984; Santos Jr, 1987).

Alguma alteração da capacidade auditiva (QUESTÃO 4) foi relatada por 4,05\% dos entrevistados. Alguns pacientes queixam-se de alteração na audição que pode ser o resultado de espasmos no tensor timpânico. Tem sido demonstrado que em algumas situações a terapia dental reduz os sintomas otológicos (Okeson, 1992).

O tamponamento auditivo (QUESTÃO 5) foi citado como uma sensação eventual por 23,24\% dos entrevistados. A obstrução dos ouvidos é um sintoma encontrado em um número significativo de casos após terapia dental. Não há base científica para justificar a relação entre a disfunção e este sintoma (Maciel, 1998).

$\mathrm{O}$ ato de apertar ou ranger os dentes (QUESTÃO 6), ou seja, o bruxismo, foi uma queixa freqüentemente relatada, sendo que $22,14 \%$ dos entrevistados o fazem habitualmente e 16, 97\% ocasionalmente. De acordo com Garcia et al (1997), esses hábitos produzem alteração no mecanismo de lubrificação das estruturas articulares, decorrente da sobrecarga nas superfícies articulares levando a alterações na biomecânica da ATM. Okeson (1992) relata que tais hábitos parafuncionais podem ser diurnos ou noturnos; e o bruxismo noturno, que ocorre com maior freqüência, geralmente não é notado. Uma das conseqüências dessa parafunção é a dor nos músculos da face, causada por cansaço ou fadiga (Siqueira, 2001).

As fraturas dentárias e de restaurações (QUESTÃO 7) foram citadas por 13, 28\% dos entrevistados. As fraturas dentárias estão associadas às forças oclusais. Dentes despolpados, com grandes restaurações e trabalhos protéticos estão sujeitos a fraturas. A fratura ocorre principalmente por trauma resultante de contato prematuro nesses dentes fragilizados.

A hipertrofia do masseter (QUESTÃO 8) foi o sintoma que obteve o maior índice de respostas negativas (92,98\%), sendo que o índice de respostas positivas foi de $6,64 \%$. A hipertrofia muscular é conseqüência da hiperatividade muscular, que está diretamente relacionada aos hábitos parafuncionais. $\mathrm{O}$ baixo índice encontrado na análise dos resultados provavelmente se deve ao fato de esse sintoma não ser muitas vezes percebido por parte dos entrevistados. Segundo Maciel (1998), a hipertrofia muscular está relacionada a distúrbios como o bruxismo e o apertamento dental. Pode trazer como conseqüência a assimetria facial e a obstrução do ducto parotídeo.

Em relação à dificuldade para dormir (QUESTÃO 9), 3,69\% dos entrevistados afirmaram apresentar tal distúrbio. Essa dificuldade está muitas vezes relacionada à ansiedade, ao estresse e à depressão. Entre as várias desordens do sono temos o bruxismo e a apnéia (Maciel, 1998).

Grande parte da população estudada (61,62\%) se considerou tensa, nervosa ou ansiosa em algum grau (QUESTÃO 10), sendo essa questão a que obteve maior índice de respostas positivas. Para Moreira et al (1998), os fatores emocionais parecem desempenhar papel significante na DTM pois podem contribuir não somente para o aparecimento dessa alteração, mas também para a sua perpetuação. 


\section{Conclusões}

Diante da análise dos resultados obtidos, julgados à luz da avaliação efetuada, pode-se concluir que:

1. Entre os estudantes analisados, 125 (46,12\%) eram portadores de DTM leve, 12 (4,42\%) moderada, nenhum apresentou DTM severa e 132 (48,70\%) estavam livres de DTM.

2. As mulheres, em relação aos homens, apresentaram maior incidência de DTM leve e moderada (53,76\% e 6,87; 35,14\% e 2,7\%, respectivamente).

3. As queixas mais freqüentes apresentadas pelos estudantes entrevistados foram tensão emocional e o hábito de apertar ou ranger os dentes.

4. O modelo de questionário empregado possibilita a estruturação de prontuário odontológico.

5. É grande a incidência de DTM e também relevante a nova especialidade Disfunção TêmporoMandibular e Dor Orofacial, bem como sua inclusão na graduação para um maior aprofundamento teórico e prático.

\section{REFERÊNCIAS}

1 ALVES, L. A. Dor orofacial - Desordens temporomandibulares. Entendendo problemas da ATM. Disponível na internet. http://members.tripod.com/ glaucialopes/io/ textos2.htm. Pesquisa em 17/03/2002.

2 GAGE, J. P.; SHAW, R. M.; MOLONEY, F. B. Collagen type in dysfunctional temporomandibular joint disks. J. Prosthet Dent., v. 74, n.5, p.517-20, 1995.

3 GARCIA, A. R.; SOUSA, V. Desordens temporomandibulares: causa de dor de cabeça e limitação da função. Rev. Assoc. Paul. Cir. Dent., v.52, n.6, p.480-486, nov./dez.
1998.

4KEERSMAEKERS, K; DEBOEVER, J. A.; VANDERBERGHE, L. Otalgia in patients with temporomandibular joint disorders. J. Prosthetic Dent., v.75, n.1, p.72-5, jan. 1996.

5 MACIEL, R. N. ATM e Dor Craniofacial: Fisiopatologia. São Paulo: Editora Santos, 2002.

6 MACIEL, R. N. Oclusão e ATM - Procedimentos clínicos. São Paulo: Editora Santos, 1998.

7 MEIRA, G. S. P. DTM x Sintomas Otológicos. Disponível na internet. http://www.aonp.org.br/fso/revista7/rev712a.htm. Pesquisa em 17/03/2002.

8 MOREIRA, M. M. S.; ALENCAR JÚNIOR, F. G. P.; BUSSADORI, C. M. M. Fatores psicológicos na etiologia da disfunção craniomandibular. Rev. Assoc. Paul. Cir. Dent., v.52, n.5, p.377-381, set./out. 1998.

9 MORESCA, R.; URIAS, D. Estudo epidemiológico dos ruídos da ATM em adultos jovens sul-brasileiros- Parte 1. JBA- Jornal Brasileiro de Oclusão, ATM \& Dor Orofacial, v.1, n.2, p.121129, abr.jun. 2001.

10 OKESON, J. P. Fundamentos de Oclusão e Desordens Temporomandibulares. 2 ed. São Paulo: Artes Médicas, 1992.

11 RAMFJORD, S.; ASH, M. M. Oclusão. 3.ed. Rio de Janeiro: Interamericana, 1984.

12 RIEDER, C. E.; MARTINOFF, J. T.; WILCOX, S. A. The prevalence of mandibular dysfunction. Part. I: Sex and age distribution of related signs and symptoms. J. Prosthet Dent., v. 50, n.1, p.81-8, 1983.

13 RIZZATTI BARBOSA, C. M.; ARANA, A. R. S.; CUNHA JR., A. C.; MORAIS, A. B. A.; GIL, I. A. Avaliação diária da dor na desordem temporomandibular: caso clínico. Rev. ABO nac., v.8, n.3, p.171-5, jun.-jul., 2000.

14 SANTOS JR., J. Oclusão: Tratamento da Sintomatologia Craniomadibular. São Paulo: Pancast, 1987.

15 SIQUEIRA, J. J. T. Dor Orofacial/ATM - Bases para o diagnóstico clínico. 2.ed. Curitiba: Editora Maio, 2001. 